Tohoku J. Exp. Med., 1993, 170, 25-34

\title{
Clinical and Pathological Investigation of Endometrial Mixed Mesodermal Tumor
}

\author{
Kоzо Іто \\ Department of Obstetrics and Gynecology, Kinki University \\ School of Medicine, Osaka-Sayama 589
}

\begin{abstract}
Iто, K. Clinical and Pathological Investigation of Endometrial Mixed Mesodermal Tumor. Tohoku J. Exp. Med., 1993, 170 (1), 25-34_Four cases of mixed mesodermal tumor (MMT) of uterine origin were histologically and cytologically studied. In the case of occurrence of rhabdomyosarcoma, the cells in the aspiration smear appeared independently and sporadically and were relatively large in size and polyhedral in shape. The cell margins were not clearly distinguishable, and the cytoplasm was non-uniformly and heavily stained light green in general except for some pale red eosinophilic areas. The swollen and elliptical nuclei had a high $\mathrm{N} / \mathrm{C}$ ratio. The chromatin showed a densely stained coarse granular pattern, and the nuclear margin was expanded. On the imprint smear, the cells contained abundant cytoplasm with amoeboid protrusions. The cytoplasm was light green in general, was filled with granules of various sizes which were non-uniformly and heavily stained. The chromatin showed coarse granulation, and a large nucleolus was observed. ——uterus; endometrial carcinoma; carcinosarcoma; cytology
\end{abstract}

In 1966, Norris et al. classified various sarcomas of uterine origin into two groups and termed the homologous sarcomas carcinosarcoma and the heterologous sarcomas MMT (mixed mesodermal tumor) (Norris and Taylor 1966; Norris et al. 1966). This classification is now generally accepted. Although few cases of MMT have been observed, their histological characteristics have been described especially in Europe and the USA (Norris and Taylor 1966; Norris et al. 1966; Kempson and Bari 1970; Chuang et al. 1970; Barwick and Livolsi 1979). Clinico-pathologic study of cases showed that MMT has a worse prognosis than endometrial carcinoma, and that this disease is more prevalent in elderly women. With the projected increase in aged people, further study of MMT, one of the malignant uterine tumors, will be of greater importance.

MMT rapidly proliferates and invades lymphatics and blood vessels to cause lymphogenous and hematogenous metastasis. Early diagnosis and appropriate treatment are thus essential. Although histodiagnosis of the endometrium is essential, accurate diagnosis prior to treatment of MMT is difficult (Chuang et al.

Received July 17, 1992; revision accepted for publication April 5, 1993.

Address for reprint; Kozo Ito, Department of Obstetrics and Gynecology, Kinki

University School of Medicine, 377-2 Ohno-Higashi, Osaka-Sayama, Osaka 589, Japan. 
1970; Barwick and Livolsi 1979). We believe that cytodiagnosis is of great help in the differentiation of this disease in the early stage. We thus studied the tumor cytologically and observed some characteristics of rhabdomyosarcoma, a constituent of MMT.

\section{Materials and Methods}

We studied four cases of MMT of uterine origin cytologically. Cells collected before treatment (by scraping of the cervix, aspiration of the uterine cavity, endocyte, or imprint smear) and ascitic cytologic samples of relapsed cases were observed using Papanicolaou, PTAH, PAS, Alcian Blue, and Giemsa staining. At least ten blocks were collected from each case and fixed in 10\% formalin to be processed for H-E, PTAH, Azan, PAS, or Alcian blue stained histological paraffin sections.

\section{RESULTS}

\section{Clinical findings}

The details are given in Table 1.

1) The age of onset ranged from 57 to 74 years (mean 65.8 years). All 4 cases showed onset after menopause.

2) One case was a non-gravida while the other three were multiparas.

3) The initial symptom was postmenopausal bleeding for all four cases.

4) Two cases were diagnosed as uterine cancer and the other two as uterine sarcoma prior to surgery. None of the four cases had any history of exposure to radiation.

\section{Macroscopic findings}

The tumor originated from the body of the uterus; it showed bolus-like proliferation into the uterine cavity in two cases, and polyp-like protrusion in the other two cases. The tumor was massive, resilient and pale yellow in color, and hemorrhage, edema, and necrosis were observed in all four cases. The border between the tumor and the circumferential myometrium was relatively clear.

\section{Histological findings}

The histological constituents of the four cases are shown in Table 2. All four cases had adenocarcinoma as the epithelial component, while three cases had rhabdomyosarcoma, two cases chondrosarcoma, and one case stromal sarcoma as the non-epithelial component. The histological appearance of the rhabdomyosarcoma is shown in Fig. 1. It comprised of hyperchromatic cells with eosinophilic cytoplasm and numerous coarse chromatin patterns. One or multiple clear nucleoli were observed. The cells showed marked pleomorphism and contained bizarre nuclei which were non-uniform in shape and showed staining characteristics of PAS-positive substance and eosinophilic globules. Fig. 2 shows cells stained with PTAH. The degree of infiltration into the uterine wall and invasion into the lymphatics and blood vessels are listed in Table 2. 


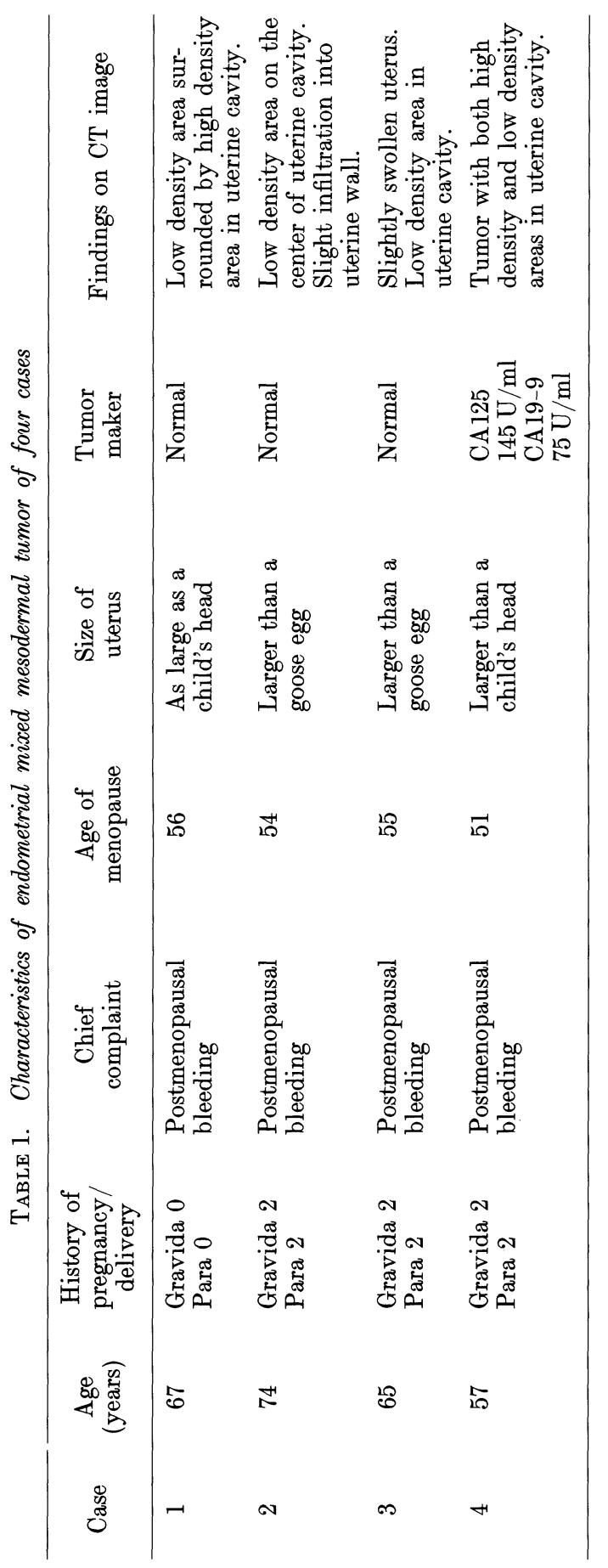




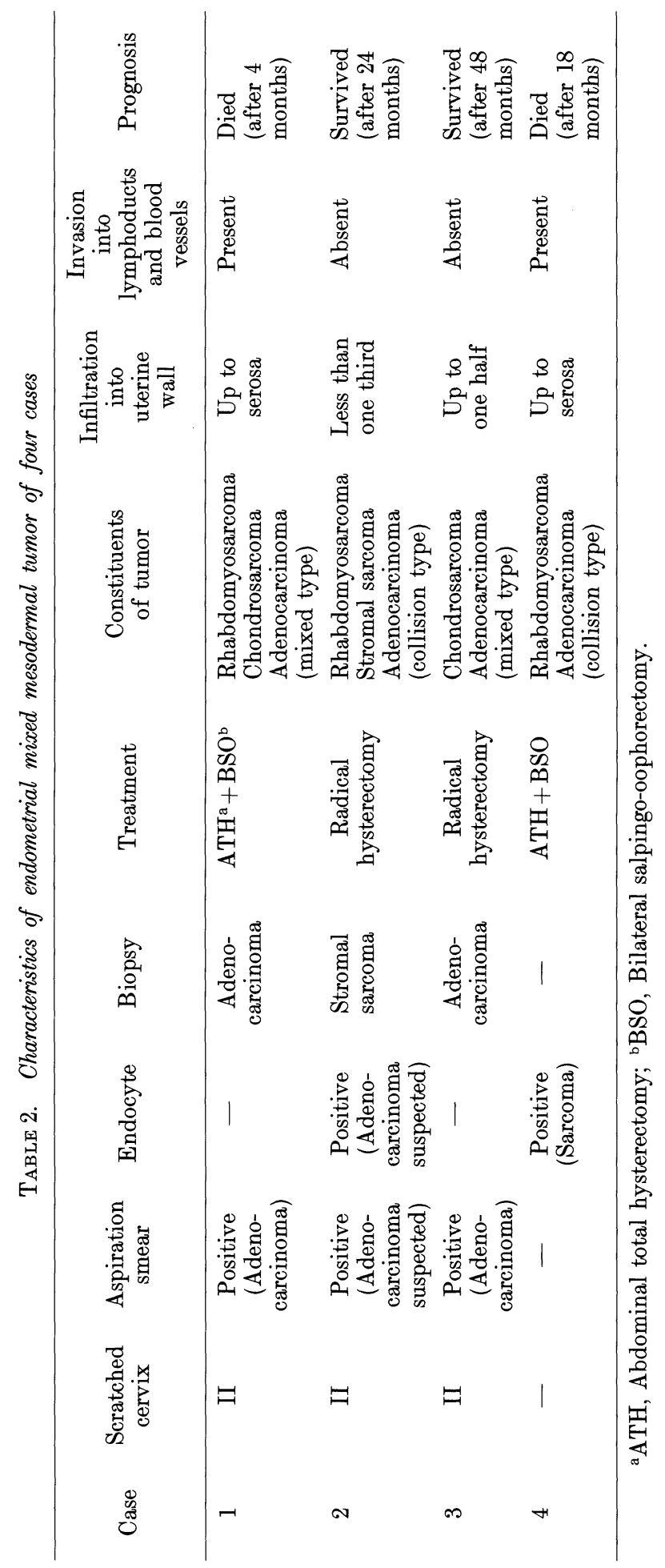




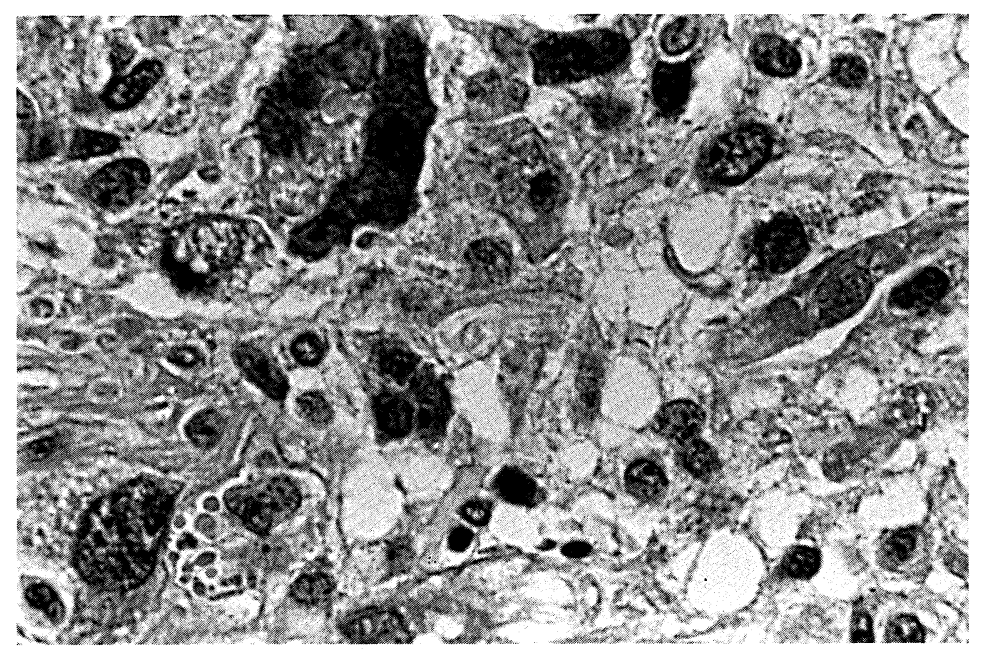

Fig. 1. Histological appearance of rhabdomyosarcoma $(\times 400)$ H.E. stain.

The sarcoma consists of circular or cone cells with abundant eosinophilic follicles. The nuclei exhibit non-uniform staining characteristics and shape, and some cells have bizarre nuclei.

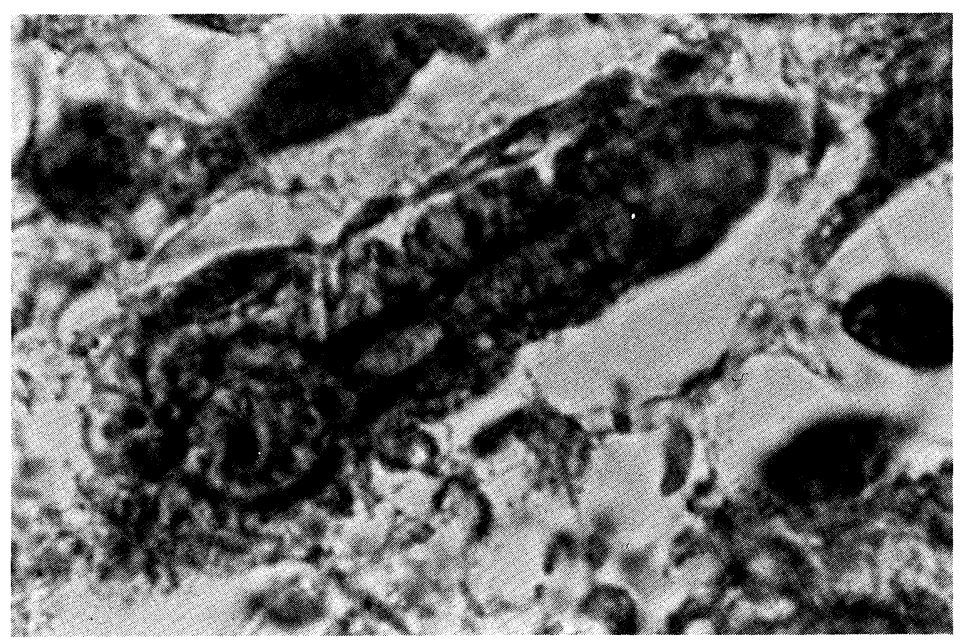

Fig. 2. Rhabdomyosarcoma stained with PTAH $(\times 1,000)$.

Cone cells with horizontal or vertical stripes. The nuclei are piled up externally.

\section{Cytological findings}

As shown in Table 2, all four cases had uterine adenocarcinoma as the epithelial component, which was easily diagnosed by aspiration or endocyte method. On the other hand, MMT, the non-epithelial component, contained 


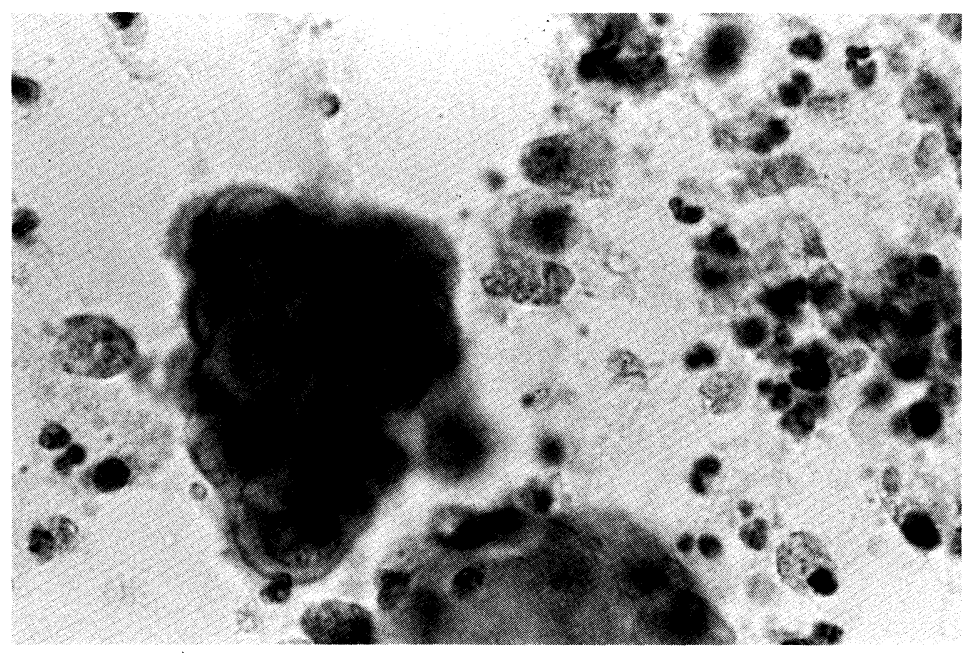

Fig. 3. Aspiration smear of adenocarcinoma cells $(\times 400)$ Pap. stain. A collection of malignant cells diagnosed as adenocarcinoma cells.

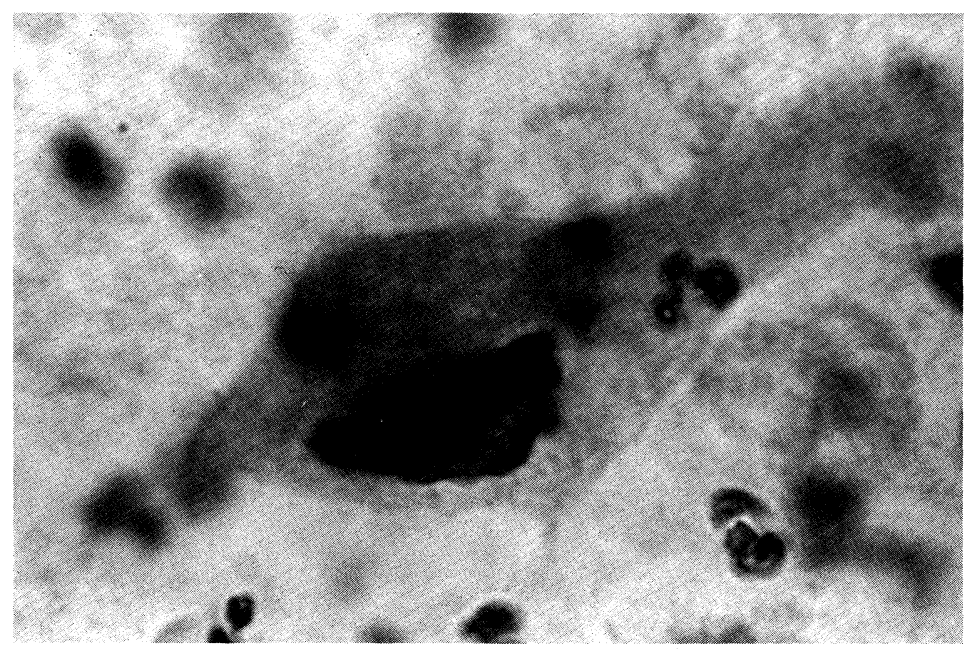

Fig. 4. Aspiration smear of rhabdomyosarcoma cells $(\times 1,000)$ Pap. stain. Rhabdomyosarcoma cells with abundant follicles and hyperchromatic nuclei of non-uniform shape.

various malignant cell types, which were not easily differentiated from each other. Fig. 3 shows a case diagnosed as adenocarcinoma on the basis of the aspiration smear. Fig. 4 shows rhabdomyosarcoma cells in the same aspiration smear, which were present independently and sporadically, and were relatively large in size and polyhedral in shape. The cell margins were not clearly distinguishable, and the cytoplasm was non-uniformly and heavily stained light green in general, except for some pale red eosinophilic areas. The swollen and elliptical nuclei had a high 


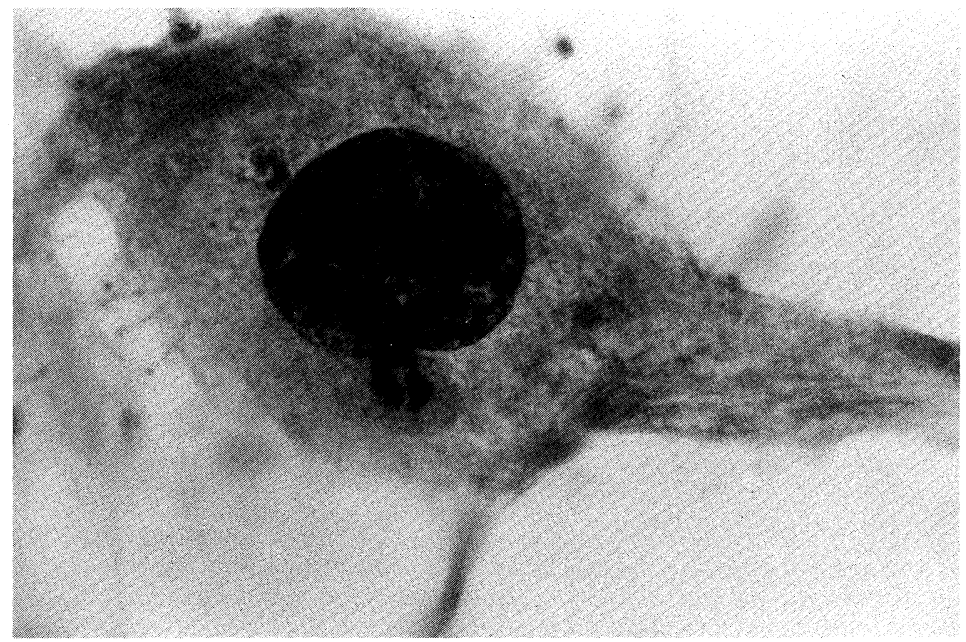

Fig. 5. Imprint smear of rhabdomyosarcoma cells $(\times 1,000)$ Pap. stain.

Rhabdomyosarcoma cells with a tadpole shape. The cytoplasm is filled with various granules and hyperchromatic nuclei are present.

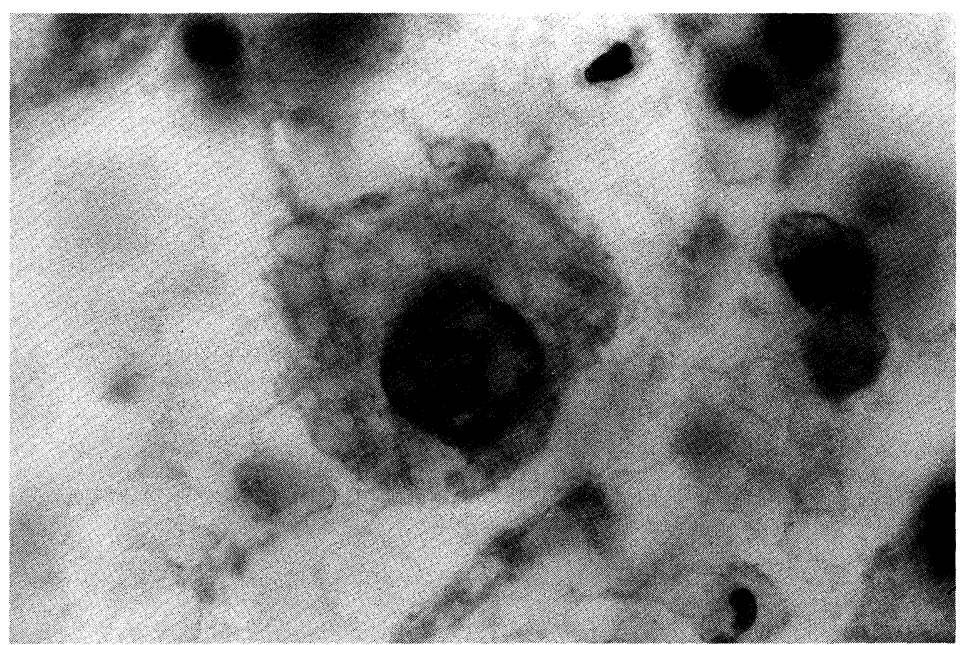

Fig. 6. Imprint smear of rhabdomyosarcoma cells $(\times 1,000)$ Pap. stain.

Findings are similar to those of Fig. 5 except for the presence of large red nucleoli.

$\mathrm{N} / \mathrm{C}$ ratio. The chromatin showed a densely stained coarse granular pattern and the nuclear margin was expanded.

Figs. 5 and 6 show the imprint smear of the tumor. The cells had abundant cytoplasm with amoeboid protrusions. The cytoplasm was non-uniformly and heavily stained light green in general, and was filled with granules of various sizes. $\begin{array}{ll}\text { The chromatin showed coarse granulation and a large nucleolus was seen. } & \text { Fig. } 7\end{array}$ 


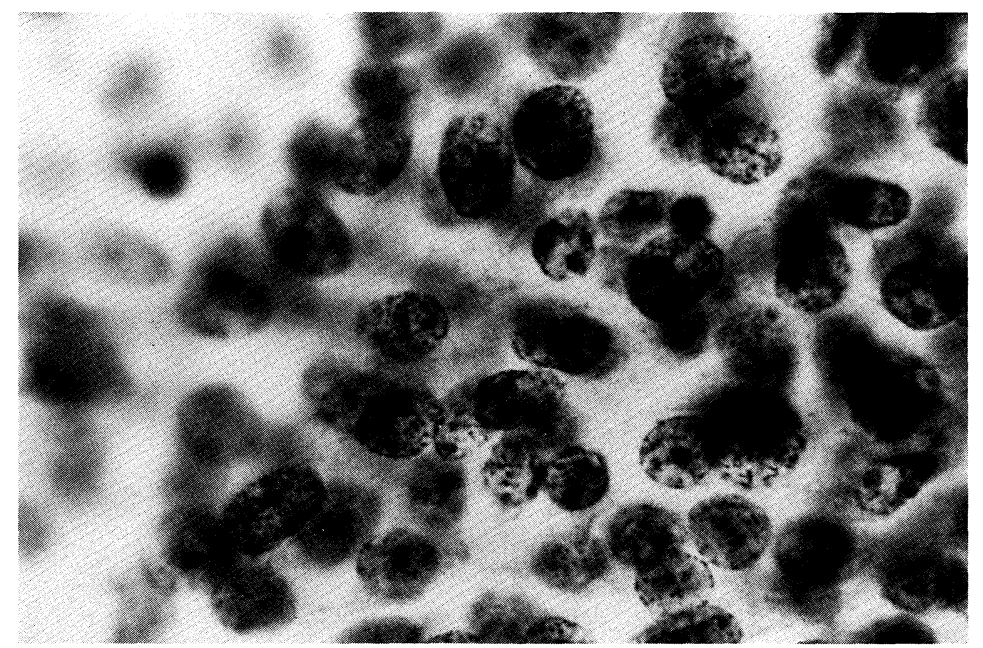

Fig. 7. Imprint smear of endometrial stromal sarcoma cells $(\times 1,000)$ Pap. stain. Stromal sarcoma cells with cytoplasm lightly stained in a lace-like shape and a localized circular nuclei.

shows the imprint smear of the endometrial stromal sarcoma of case 2 . The cells of the stromal sarcoma did not appear sporadically but formed aggregates, in contrast to the non-epithelial cells described above. The cytoplasm was stained light green in a lace-like pattern, and the cell margin was unclear. The nuclei were circular and ubiquitous, and the chromatin showed a coarse granular pattern of staining. The nuclei had slightly expanded margins and contained small multiple nucleoli. Furthermore, they were irregularly overlapping.

\section{Discussion}

In Europe and the USA, MMT comprises 1 to 2 percent of malignant uterine tumors, and has thus been described in many clinicopathologic studies, including those of Norris et al. (1966), Norris and Taylor (1966) and Chuang et al. (1970). Although there have been few cases of MMT observed in Japan, recently, clinicopathologic studies including that of Sakamoto et al. have been reported, and indicate a tendency for the disease incidence to increase (Sakamoto and Sugano 1976). With the projected increase in aged people, further study of MMT, a malignant uterine tumor, will be of great importance. In 1970, Kempson and Bari defined tumors with epithelial and non-epithelial malignant cells as malignant mixed mesodermal tumor, and further classified them into two groups: homologous type (composed of mesenchymal cells present in the normal uterus) and heterologous type (consisting of heterotopic cells that do not exist in the normal uterus). Silverberg studied two MMT cases and found no evidence of transition between cancer cells and sarcoma cells on electron microscopy. There was also no transition between cancer cells and sarcoma cells and no intermediate 
cells observed on histological study of endometrial carcinosarcoma by Rubin (1959). Based on these results, Silverberg (1971) proposed the entity of "composition tumor", in which sarcoma from Muller's duct and the surrounding mesenchyme induce cancer of the gland epithelium (Silverberg 1971). Bartsich et al. supported the theory of Muller's duct origin, that is, of combination tumor (Bartsich et al. 1967). The coexistence of both epithelial and non-epithelial malignant cells is a criterion for the cytologic diagnosis of MMT. The epithelial malignant cells are, however, more distinct, as shown in previous studies, since the cells collected depend on features of tumor proliferation such as form, existence of necrosis, and distribution of each cellular constituent. Hajdu et al. have reported that atypical malignant endometrial cells are generally observed and stromal malignant cells are also found alone or in pairs (Hajdu and Hajdu 1976). As described above, the rhabdomyosarcoma cells appear independently and sporadically and are relatively large in size and polyhedral in shape. The cell margins are not clearly distinguishable, and the cytoplasm is non-uniformly and heavily stained light green with some pale red eosinophilic areas. Furthermore, these cells appear frequently. These findings form an important basis for the diagnosis of mixed mesodermal tumor. MMT generally shows polyp-like proliferation into the body of the uterus. Biopsy of the endometrium frequently reveals no sarcoma cells but only adenocarcinoma cells, and many cases of MMT are diagnosed as uterine cancer. Alternatively, in some cases, neither sarcoma nor adenocarcinoma cells are found due to their degeneration and necrosis, which is ascribed to insufficient blood supply to the tumor due to polyp-like growth, poor circulation due to compression by the cervix, or infection by intravaginal prolapse of tumor. Thus, the tumor is often diagnosed as necrotic submucosal myoma. Cytologic studies are of great help to prevent such misdiagnosis prior to surgery. Compared with the imprint smear, many cells of the aspiration smear of the sarcoma component of the tumor are degenerated or necrotic. Accordingly, the condition of the tumor should be considered in the performance of cytodiagnosis. When the characteristic cytological findings of rhabdomyosarcoma described above are observed, the diagnosis of MMT can be made. As indicated by Howdon et al., differentiation of cells other than rhabdomyosarcoma is very difficult (Howdon et al. 1964).

Further cytological studies of MMT are required in order to achieve accurate diagnosis of MMT. Experience and accumulated cytodiagnostic data enable early diagnosis and treatment, and thereby the cure rate of the disease, which is now believed to have a poor prognosis, will be greatly improved.

\section{References}

1) Bartsich, E.G., O'Leary, J.A. \& Moore, J.G. (1967) Carcinosarcoma of the uterus A 50-year review of 32 cases (1917-1966). Obstet. Gynecol., 30, 518-523.

2) Barwick, K.W. \& Livolsi, V.A. (1979) Malignant mixed muellerian tumor of the 
uterus. A clinicopathologic assessment of 34 cases. Am. J. Surg. Pathol., 3, 125-135.

3) Chuang, J.T., Van Velden, D.J.J. \& Graham, J.B. (1970) Carcinosarcoma and mixed mesodermal tumor of the uterine corpus: Review of 49 cases. Obstet. Gynecol., 35, 769-780.

4) Hajdu, S.I. \& Hajdu, E.O. (1976) Cytopathology of Sarcomas and Other Nonepithelial Malignant Tumors. 189, Saunders Co., Philadelphia.

5) Howdon, W.H., Howdon, A., Frost, J.K. \& Woodruff, J.D. (1964) Cyto- and histopathologic correlation in mixed mesenchymal tumors of the uterus. Am. J. Obstet. Gynecol., 89, 670-679.

6) Kempson, R.L. \& Bari, W. (1970) Uterine sarcomas. Classification, diagnosis and prognosis. Hum. Pathol., 1, 331-349.

7) Norris, H.J. \& Taylor, H.B. (1966) Mesenchymal tumors of the uterus. III. A clinical and pathologic study of 31 carcinomas. Cancer, 19, 1459-1465.

8) Norris, H.J., Roth, E. \& Taylor, H.B. (1966) Mesenchymal tumors of the uterus. II. A clinical and pathologic study of 31 mixed mesodermal tumors. Obstet. Gynecol., 28, $57-63$.

9) Rubin, A. (1959) The histogenesis of carcinosarcoma (mixed mesodermal tumor) of the uterus as revealed by tissue culture studies. Am. J. Obstet. Gynecol., 77, 269-274.

10) Sakamoto, A. \& Sugano, H. (1976) Mixed mesodermal tumor of the uterine body: Relationship between histology and survival. Gann, 67, 263-273.

11) Silverberg, S.G. (1971) Malignant mixed mesodermal tumor of the uterus: An ultrastructural study. Am. J. Obstet. Gynecol., 110, 702-712. 